\title{
Die neue Wunderkur für Heroinabhängige - Dr. Zobins «Transmodulationsblockade»
}

\section{Ruedi Stohler ${ }^{a}$,}

Andreas Moldovanyi ${ }^{b}$

a PD Dr. med., LA Zentrum für Abhängigkeitserkrankungen Psychiatrische Universitätsklinik Zürich

b Dr. med., LA Polikliniken Crossline und Lifeline, Intervention/Therapie Frankental, Stadtärztlicher Dienst Zürich
Korrespondenz:

PD Dr. med. R. Stohler

Psychiatrische Universitätsklinik

Zürich

Zentrum für Abhängigkeits-

erkrankungen

Selnaustrasse 9

CH-8002 Zürich

Tel. 0442055800

Fax 0442055802

rudolf.stohler@puk.zh.ch

\section{Einleitung}

Aus Moskau kommt diesmal die frohe Botschaft. Ein Dr. Michael Zobin hat - nach jahrelangen Studien - endlich einen Weg aus der Opiatabhängigkeit entdeckt. Seit einiger Zeit werden im Suchtbereich tätige Kolleginnen und Kollegen beunruhigend häufig von Heroinabhängigen gefragt, was von der neuen Zobinschen Entzugsmethode zu halten sei. Offensichtlich wird nun auch in der Schweiz die Werbetrommel für dieses Verfahren gerührt. Dem Vernehmen nach sollen bereits acht Schweizer Patientinnen und Patienten behandelt worden sein - natürlich mit durchschlagendem Erfolg. Insgesamt seien sogar über 700 mit einer Erfolgsquote von 85\% behandelt worden. Eine weitere «therapeutische» Gruppenreise aus der Schweiz sei geplant, diesmal in Begleitung eines Teams des Schweizer Fernsehens.

\section{Die Methode}

Die neue Entzugsmethode ist geheim. In wissenschaftlichen Zeitschriften lassen sich keine einschlägigen Publikationen finden (www.ncbi.nlm. nih.gov/entrez/query.fcg; zobin m). Hingegen ist die Entzugsmethode auf verschiedenen Internetseiten beschrieben (www.zobin.ru/deutsch/ personal.htm; www.zobin-ch.com). Gemäss diesen Websites handelt es sich bei der neuen, revolutionären Methode um folgendes: «Alle Rezeptoren im Gehirn werden durch den speziellen Stoff Neuropeptid blockiert [...]. Das Aktivitätsgebiet des Rezeptors wird gänzlich vom Neuropeptid bedeckt [...]. Der Zugang zu den Gehirnressourcen ist wieder frei und die Synthese von Endorphinen beginnt allmählich wieder einzusetzen. Etwa ein Jahr nach der Behandlung rutscht das Neuropeptid wegen der Glättung des Reliefs vom Rezeptor ab. Das Gehirn fängt an, neue, gesunde Rezeptoren zu schaffen und kehrt so langsam zur Ausgangslage zurück, in der es sich vor der Drogensucht befunden hat [...].

Die Prozedur selbst bei der Blockierung der Opiatrezeptoren setzt zuallererst voraus, dass Bedingungen geschaffen werden, damit das Neu-

\section{Nouvelle cure miracle pour héroïnomanes}

Les thérapeutes à l'œuvre dans le domaine de la toxicomanie sont toujours plus interpellés par des drogués ou par leurs proches au sujet de la méthode de désintoxication mise au point par le Dr Zobin. Selon des indications figurant sur l'internet, ce médecin russe installé à Moscou est sensé avoir développé une méthode susceptible de guérir les héroïnomanes de leur dépendance avec un taux de réussite avoisinant les $\mathbf{1 0 0} \%$. Les publications scientifiques relatives au blocage de la transmodulation sont introuvables dans les banques de données habituelles comme Pubmed. Compte tenu des informations disponibles, il faut impérativement déconseiller ce traitement de 15000 francs dont les risques sont assumés par le patient.

ropeptid ungehindert zum Rezeptor gelangen kann, d.h., die Kindling-Prozesse müssen unterdrückt werden. $\mathrm{Zu}$ diesem Zweck wird eine Magnetkontur angewandt [...]. Sind jedoch nicht alle Rezeptoren blockiert, bleibt die psychische Abhängigkeit bestehen. Einzige Möglichkeit zur Kontrolle ist, am Tag nach der Neuropeptidbehandlung eine berechnete Kleinstdosis der Kontrolldroge Promedol [Pethidin, Anm. d. Verf.] zuzuführen [...] trotz Minimaldosis setzt ein totaler Atemstillstand ... beim Patienten ein. Der Patient wird natürlich sofort [...] beatmet und nach 5-7 Minuten kann der Patient wieder aus eigener Kraft atmen.»

\section{Begleitumstände}

Hoffentlich atmet er wieder, kann man da nur wünschen und noch folgende Details anfügen: Um in den Genuss der «Transmodulationsblockade» zu kommen, muss der oder die Abhängige seit mindestens drei Wochen clean sein (auf 


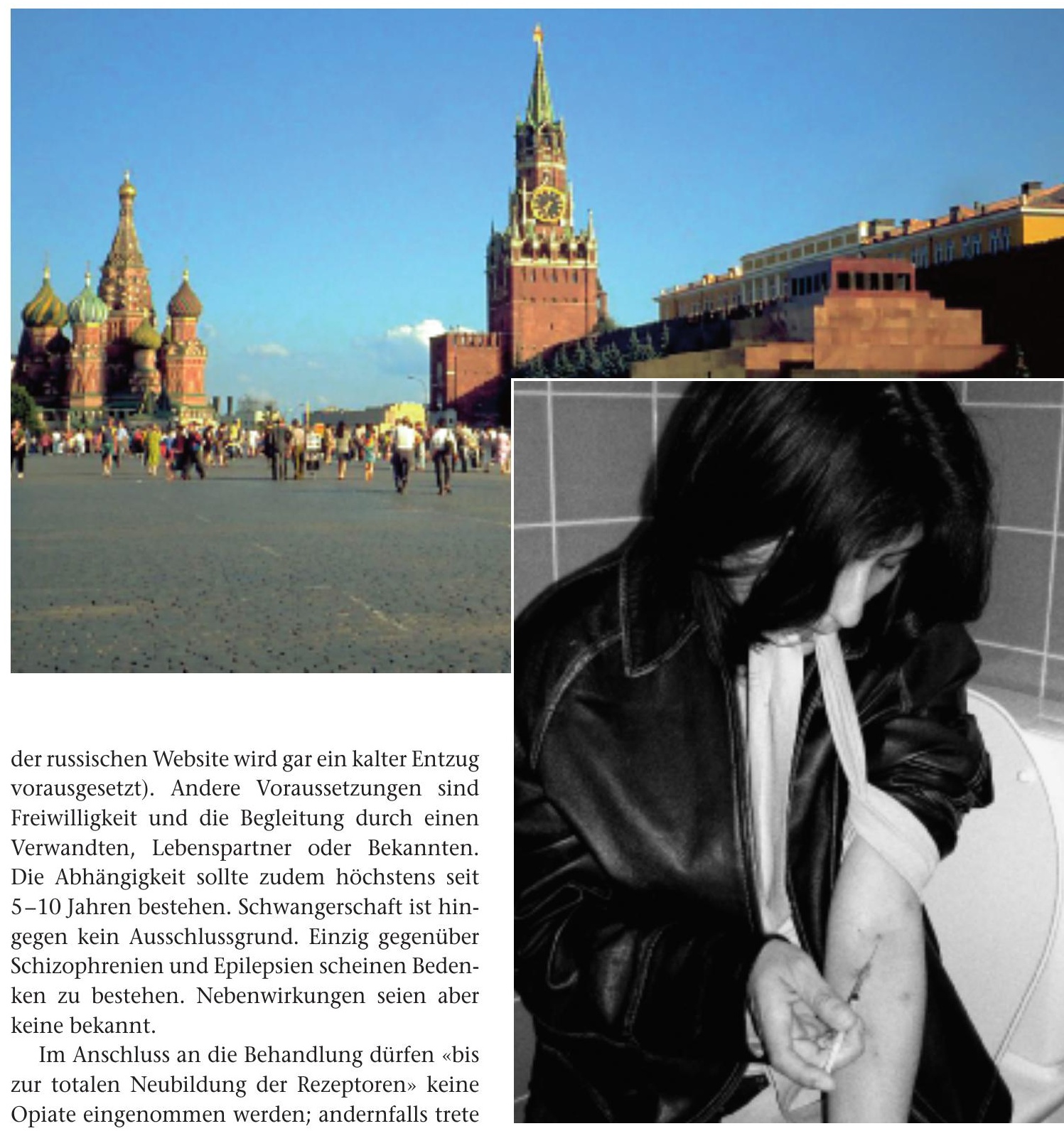
wieder ein lebensbedrohlicher Atemstillstand auf. Zumindest während dieser Zeit liege die Verantwortung für den Erfolg der Behandlung bei den Exabhängigen, die angehalten werden $\mathrm{zu}$ «einem totalen Wechsel der Umgebung», dem «Meiden von alten Freunden», einer «maximalen Beschäftigung» und zu «viel Sport». Die ganze Prozedur kostet, knapp gerechnet, Fr. 15 000.-; ohne Versicherung. Eine solche dürfte sich schwer finden lassen. Bei «Gelingen» der Behandlung sind zwei Tage für Shopping und Stadtrundfahrten einkalkuliert.

\section{Was ist von diesem Verfahren zu halten?}

Erklärtermassen soll Zobins Verfahren nur der Behandlung der «psychischen Abhängigkeit» dienen. Auch dieses eingeschränkte Behandlungsziel soll nicht erreicht, sondern sozusagen nur angeschoben werden. Die Verantwortung für

das abschliessende Gelingen liege, wie oben beschrieben, bei den Betroffenen. Direkter ausgedrückt wird somit cleanen Exabhängigen für etwa Fr. 15 000.- ein einwöchiger Aufenthalt in Moskau geboten, in dessen Verlauf ein im besten Falle nur obskures Prozedere zu erdulden ist. Im schlimmeren Falle werden sie nicht nur finanziell gerupft, sondern auch körperlich geschädigt, z. B. durch eine Gehirnhypoxie.

Wundern muss man sich leider nicht, dass es offensichtlich absurde Heilslehren immer wieder schaffen, Gläubige zu generieren. Die Verzweiflung chronisch Kranker (beispielsweise auch Querschnittgelähmter oder Tumorpatienten) und deren Umgebung ist teilweise so gross, dass das kleinste Hoffnungsfünkchen genügt, das sonst hoffentlich wachsamere Auge der Vernunft zu blenden. 